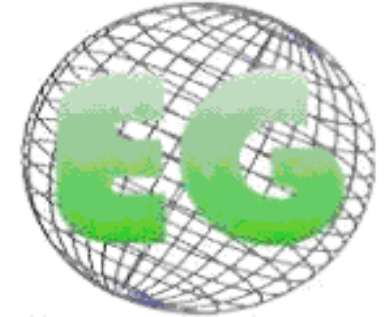

ISSN 1695-6141

$\mathrm{N}^{\circ} 21$
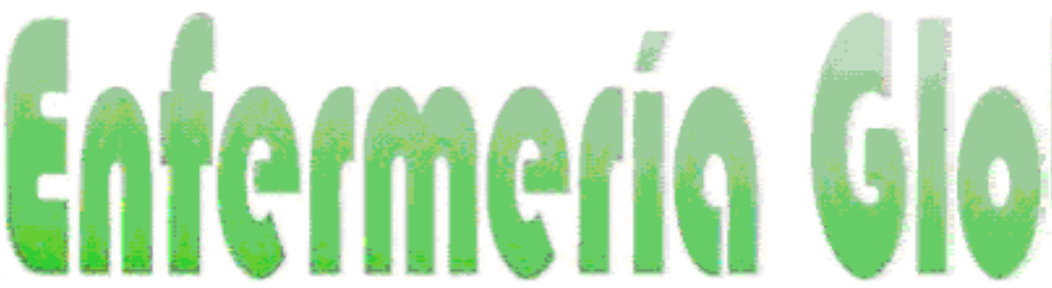

Revista electrónica trimestral de Enfermería

Enero 2011

www.um.es/egloball

\title{
ESTRATEGIAS NO FARMACOLÓGICAS PARA ALIVIAR EL DOLOR DURANTE EL PROCESO DEL PARTO
}

ESTRATÉGIAS NÃO FARMACOLÓGICAS DE ALÍVIO À DOR DURANTE O TRABALHO DE PARTO

\author{
*Sartori, AL., **Vieira, F., **Almeida, NAM., ***Bezerra, ALQ., "**Martins, CA. \\ *Mestranda em Enfermagem. **Professora Assistente. ${ }^{* * *}$ Professora Adjunta. Faculdade de Enfermagem da \\ Universidade Federal de Goiás (FEN/UFG). Brasil.
}

(Trabajo vinculado al Núcleo de Estudos em Paradigmas Assistenciais e Terapias Alternativas (NEPATA). Palabras clave: Proceso del parto, dolor de parto, obstetricia, terapias alternativas Palavras chave: trabalho de parto, dor do parto, obstetrícia, terapias alternativas

Keywords: Labour, Birth pain, Obstretics, Althernative therapies.

\section{RESUMEN}

Esta revisión de la literatura tiene como objetivo evidenciar la eficacia de las estrategias no farmacológicas para aliviar el dolor durante el proceso del parto, por medio de una investigación descriptiva exploratoria de naturaleza bibliográfica. Fueron rastreadas las producciones científicas con nivel de evidencia II, indexadas entre 1997 a febrero de 2009, en las bases de datos LILACS y Medline por medio de los descriptores "Dolor de parto", "Dolor del parto" y "Proceso del parto" asociado al "Dolor". Los seis artículos seleccionados, entre 615 producciones científicas rastreadas, fueron caracterizados y agrupados en dos categorías: Alivio del dolor durante el proceso del parto y Evidencias después de la aplicación de las estrategias no farmacológicas. Los artículos publicados entre 2003 y 2007 en seis revistas diferentes, presentaban las estrategias no farmacológicas: acupuntura, estimulación eléctrica transcutánea, técnicas de respiración y relajación. La acupuntura y la estimulación eléctrica transcutánea, presentaron eficacia aliviando el dolor durante el proceso del parto. Las otras evidencias fueron: disminución de fármacos analgésicos, de ocitócicos y también de la duración del proceso del parto. La utilización de estrategias no farmacológicas en el área obstétrica todavía no se ha concretizado. A pesar de que no se haya confirmado alivio del dolor en todos los estudios, se tienen pruebas de la obtención de otros beneficios.

\section{RESUMO}

Esta revisão sistemática da literatura objetiva evidenciar a eficácia das estratégias não farmacológicas de alívio à dor durante o trabalho de parto por meio de uma pesquisa descritiva exploratória de natureza bibliográfica. Foram rastreadas as produções científicas com nível de evidência II, indexadas entre 1997 a fevereiro de 2009 nas bases de dados LILACS e Medline por meio dos descritores "Dor de parto"; "Dor do parto" e "Trabalho de parto" associado a "Dor". Os seis 
artigos selecionados, dentre 615 produções científicas rastreadas, foram caracterizados e agrupados em duas categorias: Alívio da dor durante o trabalho de parto e Evidências após a aplicação das estratégias não farmacológicas. Os artigos, publicados entre 2003 a 2007 em seis diferentes periódicos, apresentavam as estratégias não farmacológicas: acupuntura, estimulação elétrica transcutânea e técnicas de respiração e relaxamento. A acupuntura e a estimulação elétrica transcutânea apresentaram eficácia no alívio da dor durante o trabalho de parto. As outras evidências foram: diminuição no uso de drogas analgésicas e de ocitócicos e da duração do trabalho de parto. O emprego das estratégias não farmacológicas na área obstétrica ainda não está concretizado. Embora não tenha sido evidenciado alívio da dor em todos os estudos, outros benefícios no emprego destas estratégias foram evidenciados.

\section{ABSTRACT}

The aim of this review of the literature is to show the efficiency of non pharmacological strategies in alleviating pain during labour. A descriptive, exploratory bibliographical research work is used. Scientific publications with an evidence level of II, indexed between 1997 and February 2009 were searched in the LILACS and Medline databases under the descriptors "Dolor de parto", "Dolor del parto" and "Trabajo de parto" assoociated with "Dolor" (Pain). The six selected articles from the 615 publications searched, were characterized and grouped in two categories: Alleviation of pain during labour and evidence following the application of non pharmacological strategies. The articles published between 2003 and 2007 in six different journals, presented non pharmacological strategies such as acupuncture, trans dermal electric stimulation, breathing techniques and relaxation. Acupuncture and trans skin electric stimulation were efficient in alleviating labour pains. Other findings were a decrease in painkillers, oxitocics and in duration of the birth. The use of non pharmacological strategies in obstetrics remains to be specified. Although alleviation of pain has not been confirmed in all the studies, there is proof of other benefitsd.

\section{INTRODUCCIÓN}

El dolor durante el proceso del parto es relatado desde la antigüedad, sin embargo, incluso con los recursos no farmacológicos actuales de alivio a ese síntoma todavía constituye una realidad en los servicios de obstetricia ${ }^{1}$.

Las medidas y los recursos para el control del dolor durante el parto, así como otros tipos de dolor, han sido tema de estudios y debates. En 2003 esa temática fue discutida junto a un equipo multidisciplinar en Nueva York en "The Nature and Management of Labor Pain: An Evidence-Based Symposium"2, y en el período de octubre de 2007 a septiembre de 2008 la International Sociedad for Study of Pain (IASP) lanzó una campaña "Real women, real pain", pues la proporción de mujeres afectadas por problemas de dolor, inclusive el dolor crónico, es mayor en relación al sexo masculino ${ }^{3}$.

Según la IASP el dolor se caracteriza por una experiencia sensitiva emocional desagradable asociada o relacionada con la lesión real o potencial de los tejidos ${ }^{4}$. Sin embargo, se sabe que el dolor también está influenciado por factores psicosociales y culturales, siendo de carácter individual ${ }^{1}$.

Durante el proceso del parto el dolor puede ser descrito en dos momentos, en la primera etapa, fase de dilatación, provocado por las contracciones uterinas y dilatación del cérvix. En el período expulsivo, junto a esos factores, está la presión que el feto ejerce en las estructuras pélvicas aumentando su intensidad ${ }^{5}$. 
Para evaluar la intensidad y estimar el dolor percibido se ha utilizado lo referido por la parturienta a escala analógica visual (EAV) como instrumento de medida o evaluación del dolor por su fácil aplicabilidad y comprensión6. Otras escalas, como las numéricas, de categorías de las expresiones verbales y de representación gráfica no numérica, también han ayudado a los profesionales de la salud en la evaluación del dolor.

Los avances científicos de la actualidad en el área obstétrica proporcionaron mejor comprensión de los mecanismos responsables del dolor durante el proceso del parto ${ }^{5}$, y así, la percepción del estímulo doloroso puede ser reducida por medio de medidas farmacológicas y no farmacológicas ${ }^{7}$. De entre las medidas farmacológicas es rutinario el uso de la analgesia epidural o epidural combinada con raquidiana ${ }^{8}$ y entre las medidas no farmacológicas son conocidas: terapia herbal, masajes, quiropráctica, acupuntura, aromaterapia, hidroterapia, homeopatía y aplicaciones bioeléctricas o magnéticas, deambulación, ejercicios respiratorios y musicoterapia ${ }^{1,6,8,9,10}$.

Estas terapias pueden reducir el uso de medidas alopáticas y promover sensación de bienestar para la mujer, lo que proporciona satisfacción y disminuye el stress en el momento del parto ${ }^{11}$.

No obstante, la utilización de estrategias no farmacológicas para el control del dolor durante el parto sigue estando presente en el cotidiano de las discusiones entre los profesionales, hecho que probablemente está asociado a dudas sobre la eficacia de estas técnicas ${ }^{2}$. En esa perspectiva, este estudio objetivó evidenciar la eficacia de las estrategias no farmacológicas de alivio del dolor durante el proceso del parto.

\section{METODOLOGÍA}

Investigación descriptiva exploratoria de naturaleza bibliográfica. La investigación bibliográfica proporciona el análisis de los estudios previamente realizados y, puede evidenciar aspectos que no fueron contemplados anteriormente, generando nuevas indagaciones y posiblemente nuevos estudios ${ }^{12}$. Además de ofrecer ayudas para la práctica profesional y colaborar a la mejora de la asistencia ofrecida.

Se manejaron las producciones científicas indexadas desde 1997 a febrero de 2009 en las bases de datos en Ciencias de la Salud disponibles en la Biblioteca Virtual de Saúde (BVS): Literatura Latino-Americana y del Caribe en Ciencias de la Salud (Lilacs) e Literatura Internacional em Ciências da Saúde (Medline). Las búsquedas se realizaron en el mes de febrero de 2009 por medio de los Descriptores en Ciencias de la Salud (DeCS): "Dolor del parto" o "Dolor de parto" y "Proceso del parto" asociado a "Dolor". Estos descriptores fueron escogidos con el fin de obtener el mayor número de referencias posibles para análisis.

La producción científica seleccionada obedeció a los siguientes criterios: solo artículos científicos que relatan intervenciones de las estrategias no farmacológicas durante el proceso de parto con utilización de un instrumento para evaluación del dolor, clasificado como nivel de evidencia II $^{13}$, escritos en lengua portuguesa, española, inglesa o francesa y disponibilidad del artículo científico completo en las bases de datos.

En el primer rastreo se encontraron 615 referencias, que fueron sometidas a una preselección a partir de los resúmenes y/o títulos. De este total, se seleccionaron 60 referencias, 26 no estaban disponibles online al completo, un estudio fue repetido. El restante fue excluido por tratarse de estudios de revisión o actualización, no presentaron grado de evidencia II o instrumento para evaluar el dolor, fueron realizados durante el 
período pre-natal o utilizaron enfoque cualitativo. La muestra final estuvo compuesta por seis artículos.

En el análisis descriptivo los artículos se caracterizaron por autor, año de publicación, nombre del periódico, título y tipo de estrategia no farmacológica utilizada para alivio del dolor durante el trabajo de parto. Los textos completos de los artículos fueron sometidos a una lectura cuidadosa para verificar la utilización y los efectos de las estrategias no farmacológicas durante la asistencia a la parturienta. Después, se agruparon en dos categorías: Alivio del dolor durante el trabajo de parto; Evidencias tras la aplicación de las estrategias no farmacológicas.

\section{RESULTADOS Y DISCUSIÓN}

Los datos obtenidos que caracterizan la muestra del estudio están representados en el Cuadro I.

Cuadro I. Relación de artículos seleccionados por autores, año de publicación, periódico, título y estrategia no farmacológica (ENF) utilizada.

\begin{tabular}{|c|c|c|c|c|}
\hline Autores & Año & Periódico & Título & ENF \\
\hline $\begin{array}{l}\text { Hantoushzadeh } \\
\text { S, Alhusseini N, } \\
\text { Lebaschi AH }\end{array}$ & 2007 & $\begin{array}{c}\text { Aust N Z J } \\
\text { Obstet Gynaecol }\end{array}$ & $\begin{array}{c}\text { The effects of } \\
\text { acupuncture during the } \\
\text { labour on nulliparous } \\
\text { women: a randomized } \\
\text { controlled trial }\end{array}$ & Acupuntura \\
\hline $\begin{array}{c}\text { Ziaei S, } \\
\text { Hajipour L. }\end{array}$ & 2006 & IJGO & $\begin{array}{l}\text { Effect of acupuncture on } \\
\text { labor }\end{array}$ & Acupuntura \\
\hline $\begin{array}{l}\text { Almeida NAM, } \\
\text { Sousa JT, } \\
\text { Bachion MM, } \\
\text { Silveira NA }\end{array}$ & 2005 & $\begin{array}{c}\text { Rev } \\
\text { Latino-am } \\
\text { Enfermagem }\end{array}$ & $\begin{array}{l}\text { Utilização de técnicas de } \\
\text { respiração e relaxamento } \\
\text { para alívio de dor e } \\
\text { ansiedade no processo } \\
\text { de parturição }\end{array}$ & $\begin{array}{l}\text { Técnicas de } \\
\text { respiração } \\
\text { e } \\
\text { relaxamento } \\
\text { muscular. }\end{array}$ \\
\hline $\begin{array}{l}\text { Knobel R, } \\
\text { Randuz V, } \\
\text { Carraro TE }\end{array}$ & 2005 & $\begin{array}{c}\text { Texto } \\
\text { Contexto-enferm }\end{array}$ & $\begin{array}{l}\text { Utilização de estimulação } \\
\text { elétrica transcutânea } \\
\text { para alívio da dor no } \\
\text { trabalho de parto: um } \\
\text { modo possível para o } \\
\text { cuidado à parturiente }\end{array}$ & $\begin{array}{l}\text { Estimulação } \\
\text { elétrica } \\
\text { transcutânea }\end{array}$ \\
\hline $\begin{array}{l}\text { Orange FA, } \\
\text { Amorim MMR, } \\
\text { Lima L }\end{array}$ & 2003 & $\begin{array}{c}\text { Rev. Bras. } \\
\text { Ginecol. Obstet. }\end{array}$ & $\begin{array}{l}\text { Uso da eletroestimulação } \\
\text { transcutânea para alívio } \\
\text { da dor durante o trabalho } \\
\text { de parto em uma } \\
\text { maternidade-escola: } \\
\text { ensaio clínico controlado }\end{array}$ & $\begin{array}{l}\text { Estimulação } \\
\text { elétrica } \\
\text { transcutânea }\end{array}$ \\
\hline $\begin{array}{l}\text { Nesheim Bl et } \\
\text { al. }\end{array}$ & 2003 & Clin J Pain & $\begin{array}{l}\text { Acupunture during labor } \\
\text { can reduce the use of } \\
\text { meperidine: a controlled } \\
\text { clinical study }\end{array}$ & Acupuntura \\
\hline
\end{tabular}

Los seis artículos representados arriba fueron publicados en seis periódicos diferentes, tres nacionales y tres internacionales, en los idiomas: inglés y portugués. 
Como esta revisión tuvo por objetivo demostrar la eficacia de las estrategias no farmacológicas, se seleccionaron solo artículos que presentaran nivel de evidencia II, por tratarse de estudios controlados posibles de reproducir y de metodología rigurosa, los cuales son indicados para estudios de tratamiento y prevención.

El período de las publicaciones varió entre los años de 2003 a 2007, aunque las discusiones acerca de la inserción social femenina y de la creación de políticas de atención a la salud de la mujer, principalmente durante el período gestacional, surgieron en la década de los $80^{14}$.

Así, el movimiento de humanización de la asistencia al parto a nivel mundial pudo influir en la busca de estas medidas, pues promover alivio al dolor durante el parto está íntimamente relacionado con la garantía de seguridad a la parturienta en la vivencia del proceso doloroso, y consecuentemente con un parto saludable. La asistencia prestada a la gestante y parturienta debe tener en cuenta aspectos humanistas que incorporen cuestiones sociales, culturales y económicas de esta mujer y su familia ${ }^{15}$.

Aunque sea una recomendación de la Organización Mundial de la Salud, la implementación de estrategias no farmacológicas para aliviar las incomodidades derivadas del dolor durante el proceso del parto, la adhesión a esta práctica ha estado influida por la filosofía de la institución de atención al parto ${ }^{8}{ }^{16}$. En países europeos como Francia y Dinamarca, las gestantes son estimuladas al parto natural y a recibir las prácticas no farmacológicas de alivio del dolor ${ }^{8}$.

El establecimiento de tales medidas en esos servicios puede optimizar la asistencia ofrecida a la parturienta, así como a la gestante durante el período pre-natal como forma de humanizar los servicios de salud.

En Brasil el Programa de Humanización en el Pre-Natal y Nacimiento elaborado en 2000 por el Ministerio de Salud, además de incentivar la práctica del parto normal sin intervenciones, garantiza también una asistencia humanizada adecuada a la gestante durante el período pre-natal, proceso del parto, parto y puerperio, lo que además de promover la autonomía de la mujer permite también que ella pueda experienciar ese proceso de forma menos traumática y desagradable ${ }^{7,17}$.

A partir del análisis de los artículos y los beneficios encontrados en la aplicación de las estrategias no farmacológicas se realizó la categorización temática presentada a continuación.

\section{Alivio del dolor durante el proceso de parto}

Los artículos seleccionados para evidenciar la eficacia del alivio del dolor durante el proceso del parto utilizaron las estrategias: acupuntura, estimulación eléctrica transcutánea y ejercicios respiratorios y de relajación muscular. Estas evidencias se describen a continuación.

La acupuntura se describe en tres artículos encontrados, pero solo dos presentaron resultados sobre la aplicación de esa estrategia. El otro solamente compara el uso de meperidina en las parturientas que recibieron acupuntura y fue analizado por ser este un opióide utilizado en la obstetricia como analgésico del dolor en la parturienta. 
Al inicio de las sesiones el score de dolor fue significativo y mayor en las pacientes del grupo que recibieron acupuntura (grupo intervención) que el score encontrado en las parturientas del grupo control. A las dos horas del inicio de las sesiones el score del dolor en el grupo intervención, 56.51 (52,63-60,39), fue significativamente menor que el encontrado en el grupo control, $69.61(65.99-73.84)(p<0.01)$. Los puntos utilizados para la inserción de las agujas correspondían al intestino grueso (LI 4), vejiga (UB 32 e 60), bazo (S 6), estómago (ST 36), vesícula biliar (GB 34) y corazón (HT 7) ${ }^{18}$. Sin embargo, en el estudio que utilizó más allá de los puntos LI 4 y ST 36 los puntos GV 20, LI 3, SP 6, CV 2 y 3 y Yangtang no se evidenció alivio del dolor tras dos horas de inserción de las agujas ${ }^{19}$.

Aunque los dos estudios tenían características semejantes en cuanto a los puntos escogidos, criterios de inclusión e instrumento para evaluación del dolor (EAV), que varía de 1 a 10, el número de participantes fue mayor en el primer estudio presentado, siendo de 74 participantes en el grupo de intervención y 70 grupo control, en los que las agujas fueron insertadas en puntos usados para aplicación de soluciones inyectables. El otro estudio analizado constaba de 90 participantes colocadas en tres grupos distintos de igual número, siendo un grupo de aplicación de acupuntura, otro de acupuntura pretend, que utilizó puntos, normalmente usados para aplicación de drogas inyectables y un tercer grupo de control.

La aplicación de las técnicas respiratorias y de relajación de Dick Read y Fernand Lamaze tampoco mostró alivio del dolor durante el parto. La intensidad de dolor referida a través de la EAV aumentó de acuerdo con la progresión de la dilatación cervical, no presentando diferencia estadísticamente significativa en ninguna de las fases, fase latente $(p=0.21)$, fase activa $(p=0.11)$ y fase de transición $(p=0.49)$. El score de dolor en el grupo experimental fue mayor del evidenciado en el grupo control en todas las fases, excepto en la fase de transición en que la puntuación máxima de la EAV fue referida para ambos grupos ${ }^{6}$.

La aplicación de la estimulación eléctrica transcutánea (EET) en dos grupos de 11 participantes cada uno, sin aplicación de electrodos en la región paravertebral a nivel de la décima vértebra torácica ( $(\mathrm{T} 10 / \mathrm{L}$ 1) y de la segunda vértebra sacra (S 2) no presentó resultado significativo en el alivio del dolor durante el desarrollo del parto cuando fue evaluada por medio de la EAV $(p=0.86)^{8}$.

En otro estudio, acompañando cuatro grupos de 10 participantes cada uno, se comparó el uso de dos modelos de electrodos para alivio del dolor, Placa y Silver Spike Point (SPP), con sus respectivos grupos control. La media de dolor encontrada fue de $8,5 \pm 1,5$, evidenciándose diferencia estadísticamente significativa entre el grupo control y grupo en tratamiento con electrodos modelo SPP después de $10(p=0.005), 30(p=0.001), 60$ $(p=0.007)$ y 120 minutos $(p=0.02)$, y solamente después de 10 minutos de estimulación usando electrodos del tipo Placa $(p=0.04)^{20}$.

En este último estudio, los puntos escogidos para aplicación de los electrodos fueron primero y segundo orificios sacro (B 31 y 32), puntos de estimulación diferentes de los descritos en el primer estudio.

\section{Evidencias después de la aplicación de las estrategias no farmacológicas}

Se evidenciaron otros beneficios derivados de la aplicación de las estrategias no farmacológicas que merecen ser abordados en esta revisión, los cuales están representados en las subcategorías mostradas a continuación. 
Uso de drogas analgésicas.

El uso de meperidina se comparó con la aplicación de acupuntura. Entre las parturientas que no recibieron acupuntura, 37\% utilizaron meperidina; en cuanto al grupo en que la técnica fue aplicada, $11 \%$ necesitó la administración de este opióide, resultado considerado estadísticamente significativo $(p<0.0001)^{21}$.

La proporción de mujeres que necesitaron de drogas analgésicas fue menor entre las participantes que utilizaron EET, siendo estadísticamente significante el uso del modelo Silver Spike Point $(\mathrm{p}<0.02)$. En este análisis, para evitar efectos confundidores, los autores excluyeron a las parturientas que precisaron de cesárea ${ }^{20}$.

Otro beneficio interesante encontrado fue el tiempo entre la evaluación del dolor y la administración de analgesia combinada. Cuando el score del dolor es mayor o igual a 6 en la EAV estaba indicada la raquianestesia asociada a epidural y el tiempo necesario para esa indicación fue mayor para el grupo que recibió aplicación de la EET, media de 90 minutos, en cuanto al grupo control, la media de instalación de la analgesia combinada fue de 30 minutos ${ }^{8}$.

\section{Administración de ocitócicos}

El uso de la acupuntura en mujeres con dilatación $\geq 4 \mathrm{~cm}$ y presencia de tres contracciones en el período de 40 segundos y agujas insertadas en los puntos LI4, UB32, UB60, SP6, ST36, LIV3, GB34, HT7 disminuyó las dosis de ocitocina administradas $(p=0.001)^{18}$.

Un segundo estudio también evidenció que la proporción de mujeres que necesitaron de ocitocina para aumentar las contracciones durante el desarrollo del parto fue menor entre aquellas que recibieron esa ENF si comparamos con los grupos acupuntura pretend y control, siendo de $50 \%, 76,6 \%$ e $76,6 \%$, respectivamente $(p=0.03)^{19}$.

\section{Duración del proceso del parto}

El proceso del parto se inicia con la fase latente, en la cual el patrón de las contracciones y el proceso doloroso son menores. La duración de esta fase presentó diferencia estadísticamente significativa al ser implementadas técnicas de relajación muscular y respiratorias, observándose una media de $84,7 \pm 37,10$ minutos para el grupo control y grupo experimental de 145,26 $\pm 96,57,(p=0.01)^{6}$.

Después de la fase latente, se inicia la fase activa, que corresponde al aumento de las contracciones uterinas, dilatación cervical y descenso del feto por la pelvis materna. En parturientas que recibieron acupuntura, la duración de la fase activa del proceso de parto fuei descrita significativamente menor $(p<0.01)$, teniendo media de 3.41 (3.06-3.77) horas y en el grupo control de 4.45 (4.06-4.83) horas $^{18}$.

\section{CONSIDERACIONES FINALES}

El análisis de los estudios publicados evidenció que solo tres estrategias no farmacológicas fueron aplicadas de acuerdo con el nivel de evidencia II. Otras estratégias no farmacológicas son descritas en la literatura, aunque sus formas de aplicación no mostraron nivel de evidencia significante en la práctica. Se percibe la necessidad de la realización de ensayos 
clínicos controlados a fin de que la eficacia de otras estrategias, tales como crioterapia, deambulación, musicoterapia y otras, sean validadas e incorporadas en la asistencia obstétrica.

Con relación al alivio del dolor, no todas las estrategias no farmacológicas fueron eficaces, mas disminuyeron el uso de drogas analgésicas y la administración de ocitocina en las parturientas. Además de esto, otro aspecto interesante es el que no se relataron eventos adversos perjudiciales para la parturienta y el feto.

Se acreditan resultados positivos en la aplicación de las estrategias no farmacológicas durante el proceso de parto que pueden ser intensificados si estas son asociadas e implementadas durante el acompañamiento pre-natal. En este período, la gestante podrá familiarizarse con diferentes estrategias que serán propuestas por la unidad de asistencia al parto y también por medio de la comprensión de la aplicación de las mismas y por la opción de escogida del método que mejor se adapte. Así como el profesional de salud podrá establecer vínculo de mayor aproximación con la gestante, favoreciendo la relación cliente/profesional.

De esta forma, la garantía de control del dolor de la gestante durante el proceso de parto, por medio de estrategias no farmacológicas que presentan evidencia científica de eficacia, favorecerá una asistencia obstétrica humanizada con la promoción de seguridad y de calidad.

\section{REFERENCIAS}

1. Mamede FV, Almeida AM, Souza L, Mamede MV. A dor durante o trabalho de parto: 0 efeito da deambulação. Rev Latino-am Enfermagem. 2007; 15(6):1157-62. Disponível em: http://www.scielo.br/pdf/rlae/v15n6/pt 15.pdf.

2. Young D. The nature and management of labor pain: what is the evidence? Birth. 2001; 28(3):149-51. Disponível em: http://www.blackwellsynergy.com/action/showPdf?submitPDF=Full+Text+PDF+\%2826+KB\%29\&doi=10.1046\%2 Fj.1523-536x.2001.00149.x.

3. Jensen TS. Real women, real pain. 2008. Disponível em: http://www.iasppain.org/AM/Template.cfm?Section=Real Women Real Pain\&Template=/CM/HTMLDisplay. cfm\&ContentID=4629>.

4. Merskey H, Bogduk N. Classification of chronic pain. Seatle: IAPS Press [online]. 1994. Disponível em:

Section=Pain_Definitions\&Template=/CM/HTMLDisplay.cfm\&ContentID=1728.

5. Montenegro CAB, Rezende Filho J. Obstetrícia Fundamental. 11 ed. Rio de Janeiro: Guanabara \& Koogan; 2008.

6. Almeida NAM, Sousa JT, Bachion MM, Silveira NA. Utilização de técnicas de respiração e relaxamento para alívio de dor e ansiedade no processo de parturição. Rev Latino-am Enfermagem. 2005; 13(1):52-8. Disponível em: http://www.scielo.br/pdf/rlae/v13n1/v13n1a09.pdf.

7. Davim RMB, Torres GV, Melo ES. Estratégias não farmacológicas no alívio da dor durante o trabalho de parto: pré-teste de um instrumento. Rev Latino-am Enfermagem. 2007; 15(6):1150-6. Disponível em: http://www.scielo.br/pdf/rlae/v15n6/pt 14.pdf.

8. Orange FA.,Amorim MMR, Lima L. Uso da eletroestimulação transcutânea para alívio da dor durante o trabalho de parto em uma Maternidade-escola: ensaio clínico controlado. Rev Bras Ginecol Obstet. 2003; 25(1):45-52. Disponível em: http://www.scielo.br/pdf/rbgo/v25n1/a07v25n1.pdf. 
9. Sidorenko VN. Clinical application of medical resonance therapy music in high-risk pregnancies. Integr Physiol Behav Sci. 2000; 35(3):199-207. Disponível em: http://www.springerlink.com/content/g2k377w2t0377810/fulltext.pdf.

10. Browing CA. Using music during childbirth. Birth. 2004; 27(4):272-6. Disponível em: http://www.blackwell-synergy.com/doi/full/10.1046/j.1523-536x.2000.00272.x.

11. Simkin P, Bolding A. Update on nonpharmacologic approaches to relieve labor pain and prevent suffering. Journal of Midwifery \& Women's Health. 2004; 49(6):489-504. Disponível em:

12. Marconi MA, Lakatos EM. Fundamentos de metodologia científica. 6st ed. São Paulo: Atlas. 2007.

13. Galvão CM. Níveis de evidência. Acta Paulista de Enfermagem. 06; 19(2): V. Disponível em: http://www.scielo.br/pdf/ape/v19n2/a01v19n2.pdf

14. Griboski RA; Guilhem, D. Mulheres e profissionais de saúde: o imaginário cultural na humanização ao parto e nascimento. Texto \& Contexto Enferm. 2006; 15(1):107-14. Disponível em: http://www.scielo.br/pdf/tce/v15n1/a13v15n1.pdf

15. Silva LR, Christoffel MM, Souza KV. História, conquistas e perspectivas no cuidado à mulher e à criança. Texto e Contexto Enferm. 2005; 14(4):585-93. Disponível em: http://www.scielo.br/pdf/tce/v14n4/a16v14n4.pdf

16. Organização Mundial de Saúde. Saúde Materna e Neonatal/ Unidade de Maternidade Segura, Saúde Reprodutiva e da Família. Assistência ao Parto Normal: Um Guia Prático. Genebra - Suiça. Grupo Técnico de Tradução. Brasília: Ministério da Saúde. 1996.

17. Brasil. Pré-natal e Puerpério: atenção qualificada e humanizada - manual técnico/Ministério da Saúde, Secretaria de atenção à saúde, Departamento de ações programáticas estratégicas. Brasília: Ministério da Saúde. 2005.

18. Hantoushzadeh $\mathrm{S}$, Alhusseini N, Lebaschi AH. The effects of acupuncture during labour on nulliparous women: A randomised controlled trial. Aust N Z J Obstet Gynaecol. 2007; 47:26-30. Disponível em: http://www.blackwell-synergy.com/doi/full/10.1111/j.1479828X.2006.00674.X.

19. Ziaei S. Hajipour L. Effect of acupuncture on labor. International Journal of Gynecology and Obstetrics. 2006; 92:71-2. Disponível em: http://www.sciencedirect.com/science? ob=Mlmg\& imagekey=B6T7M-4HDGBNT-5-

$1 \&$ cdi $=5062 \&$ user $=686368 \&$ orig=browse \& coverDate $=01 \% 2 F 31 \% 2 F 2006 \&$ sk $=9990799$ 98\&view $=$ c\&wchp $=$ dGLbVzb-

zSkzS\&md5=16f48ab64c84b246e9803d8eb0a7a72e\&ie=/sdarticle.pdf

20. Knobel R, Randuz V, Carraro TE. Utilização de estimulação elétrica transcutânea para alívio da dor no trabalho de parto: um modo possível para o cuidado à parturiente. Texto \& Contexto Enferm [online]. 2005 [cited 2008 jun 09];14(2):1-14. Available from: http://www.textoecontexto.ufsc.br/viewissue.php?id=13>.

21. Nesheim BI, Kinge R, Berg B, Alfredsson B, Allgot E, Hove G et al. Acupuncture during labor can reduce the use of meperidine: a controlled clinical study. The Clinical Journal of Pain. 2003; 19:187-191. Disponível em: http://www.cebp.nl/vault public/filesystem/?ID=2243 